100 c.c.; add litmus and neutralize cautiously with dilute sulphuric acid. Neutralize the slight excess of acid with barium carbonate. Filter the solution, whsh the filter, and evaporate the liquor to a syrupy consistency.

As has already been said the glycerine so obtained is not pure. Treat the contents of the capsile with strong alcohol; a large quantity of salts is thrown down. The alcoholic solution is filtered through a small filter, previously moistened with alcohol, and the filtrate is collected in a tared platinum or porcelain dish. Evaporate the alcohol on the water bath and dry in the air bath above $100^{\circ} \mathrm{C}$. to constant weight. After noting this, heat the glycerine, and burn it. The weight of the ash is deducted from the total weight; the difference is glycerine. The percentage of ash compared with the total percentage of glycerine is by itself an important factor, as glycerine derived by this mode of treament from pure butter gives $5 \%$ of ash, whereas the glycerine of oleomargarine and other similar fats leaves only from 0.3 to $0.6 \%$.

By this method I have found, after deduction of said ash,23.75\% glycerine in pure butter; $7 \%$ glycerine in oleomargarine.

\title{
DETERMINATION OF METALLIC ZINC IN ZINC DUST.
}

By Morton Liebschütz.

Fresenius has given a method based on the decomposition of acidulated water by the metallic zinc that is one of the constituents of zine dust or tutty. The hydrogen evolved is converted in water, the weight of which answers to a definite amount of zinc. This method requires all the apparatus of an organic analysis; and althongh the results are accurate the daily increasing use of this zine dust, requires that samples may be tested quickly, or compared one with the other, without loss of time and without necessitating costly manipulations.* The following method will, I think, answer the purpose when a very close estimation is not required :

Weigh out about $1 \mathrm{grm}$. of the sample, after removing metallic iron with a magnet, moisten the sample, in a beaker, with alcohol; stir with a small platinum spatula, or a piece of platinum foil, and,

*The occlusion of hydrogen by zinc dust recently noted by $G$. Williams, adds a new difflculty in the use of Fresenius' process. 
while stirring, pour at once into the beaker a warm neutral solution of cupric sulphate ( 5 grms. sulphate to 1 grm. of zinc dust). Digest for a few minutes, acidulate with a few drops of dilute sulphuric acid, to remove metallic oxides, allow the divided metallic copper to settle, and decant the solution containing the excess of sulphate used upon a small filter; wash several times by decantation with tepid water, always pouring the washings over the filter until no copper cun be detected in the filtrate with ferrocyanide.

Dissolve the divided copper in nitric acid, burn the small filter with its contents, and dissolve in a few drops of nitric acid. Put the two solutions into a graduated flask, complete the volume with water and titrate with a standard solution of potassium cyanide using an aliquot portion of the copper solution. The weight of the copper multiplied by $1 ; 031$ gives the metallic zinc.

As lead occurs of ten, and perhaps always in zinc dust, and as its presence is troublesome in the copper titration, it is preferable to treat the two nitric acid solutions, evaporated together to a small volume with sulphuric acid and to throw down lead in the form of sulphate. Collect the lead sulphate on a small filter. The filtrate free from the lead is collected in a graduated flask, and the washings of the filter are used to complete the volume. By so doing we determine also, without changing the method of analysis, the foreign metals of the zine dust.

Although pure zinc as finely divided as it is in zinc dust could not be obtained, the results with very finely filed, pure zine gave results varying from 95 to $98 \%$. It is to be supposed that at least 99\% would be found in pure zinc if it were as finely divided as it is in zine dust.

Various samples of zinc dusts have shown 85 to $98 \%$ of metallic zinc. A sample represented by the seller to contain $99.5 \%$ of metallic zinc was found to contain. more than $1 \%$ of metallic lead; and the yield in zinc, even supposing that $98 \%$ only of the true

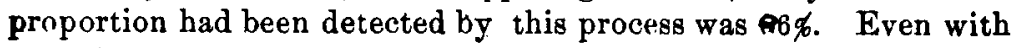
96\% of pure zinc, however, a zinc dust would be a creditable product and the claim of 99.5 is preposterous. 